\title{
Using Acrylamide Based Photopolymers for Fabrication of Holographic Optical Elements in Solar Energy Applications
}

\author{
Hoda Akbari \\ Technological University Dublin, hodaakbari@gmail.com \\ Izabela Naydenova \\ Technological University Dublin, izabela.naydenova@tudublin.ie \\ Suzanne Martin \\ Technological University Dublin, suzanne.martin@tudublin.ie
}

Follow this and additional works at: https://arrow.tudublin.ie/scschphyart

Part of the Optics Commons, and the Other Physics Commons

\section{Recommended Citation}

Hoda Akbari, Izabela Naydenova, and Suzanne Martin, "Using acrylamide-based photopolymers for fabrication of holographic optical elements in solar energy applications," Appl. Opt. 53, 1343-1353 (2014). doi:10.1364/A0.53.001343

This Article is brought to you for free and open access by the School of Physics \& Clinical \& Optometric Science at ARROW@TU Dublin. It has been accepted for inclusion in Articles by an authorized administrator of ARROW@TU Dublin. For more information, please contact arrow.admin@tudublin.ie, aisling.coyne@tudublin.ie, gerard.connolly@tudublin.ie.

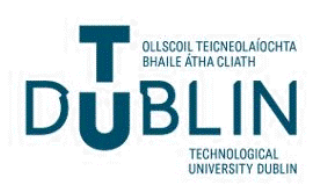




\title{
Using acrylamide based photopolymers for fabrication of holographic optical elements in solar energy applications
}

\author{
Hoda Akbari, Izabela Naydenova, Suzanne Martin * \\ Centre for Industrial and Engineering Optics, Schools of Physics, College of Science and Health, Dublin \\ Institute of Technology, Dublin 8, Ireland \\ *Corresponding email:suzanne.martin@dit.ie
}

\begin{abstract}
A holographic device is under development that aims to improve light collection in solar cells. The aim is to explore the potential of using photopolymer Holographic Optical Elements (HOE) to collect light from a moving source, such as the sun, and re-direct it for concentration by a holographic lens.. A working range of 45 degrees is targeted for such a device to be useful in solar applications without tracking. A photopolymer HOE is capable of efficiently re-directing light, but the angular selectivity of a single grating is usually of the order of one degree at the thicknesses required for high efficiency. The challenge here is to increase the angular and wavelength range of the gratings so that a reasonable number may be multiplexed and/or combined to provide a device that can concentrate light incident from a large range of angles. In this paper low spatial frequency holographic recording is explored in order to increase the angular and wavelength range of an individual grating. Ultimately, a combination of gratings will be used so that a broad range of angles of incidence are accepted. A design is proposed for the combination of such elements into a holographic solar collector. The first step in achieving this is optimization of recording at low spatial frequency. This requires a photopolymer material with unique properties, such as a fast monomer diffusion rate. This paper reports results on the efficiency of holograms recorded in an acrylamide based photopolymer at low spatial frequencies (100, 200 and $300 \mathrm{l} / \mathrm{mm}$ ). The diffraction efficiency and angular selectivity of recorded holograms have been studied for various photopolymer layer thicknesses and different intensities of the recording beams. A diffraction efficiency of over $80 \%$ was achieved at a spatial frequency of $200 \mathrm{l} / \mathrm{mm}$. The optimum intensity of recording at this spatial frequency was found to be $1 \mathrm{~mW} / \mathrm{cm}^{2}$. Individual gratings and focusing elements with high efficiency and FWHM angles of $3^{\circ}$ are experimentally demonstrated.
\end{abstract}

OCIS codes: $090.0090,090.2890$,

\section{INTRODUCTION}

Diffractive optical elements have the potential to collect light from a large area and focus or re-direct the light onto a smaller area, which has obvious advantages for solar applications. Commercially available diffractive elements tend to be surface holograms produced by photolithography and are not ideally suited to solar applications. For the collection of light from a moving source such as the sun, slanted, highly efficient gratings are needed to re-direct light that is incident from many directions into one location where it can be concentrated and converted. Diffractive elements fabricated using holographic processes (Holographic Optical Elements) are well suited to this task. However, their high angular selectivity is a problem.

The key advantage of HOEs for solar applications is their ability to efficiently diffract light through a large angle, but they also offer other features that can be used to advantage such as potential for multiplexed gratings (multiple gratings occupying the same layer), variable wavelength and angular selectivity and flexible design. HOEs are thin, flat and lightweight optical elements, which make them attractive in many applications. They can function as gratings, lenses, beam splitters, spectral filters, shear elements, achromats, mirrors, birefringent elements, and multi-function elements. They can be used, for example, in optical sensors [1-7], fibre-optics [8-11], optical scanners [12-14], optical disk pick-up heads [15-16] as well as holographic concentrators [17-19].

In order to design a holographic solar concentrator, the position of the sun at different times of the day and year needs to be considered. Ludman suggested in 1982 that HOEs could be used in solar applications because they 
have the capability to redirect, concentrate or block the incident light [20]. A number of other researchers have demonstrated novel designs over the years, for example Zhang et al. [21] proposed the use of HOEs to offer 1-D coarse tracking instead of 2-D tracking reducing the cost of the system. Ren et al., 2007 proposed a system which concentrates the visible spectrum over a 60 degree angular variation by using holographic Fresnel lenses [22], Sam et al., 2007, proposed a design based on multi-beam reflection HOE for light concentration [23].

In this paper we focus on the potential for photopolymer HOEs in redirecting the light to Photovoltaic (PV) devices. We propose a design that exploits the particular characteristics of an acrylamide based photopolymer in order to create a device that collects light from a range of angles and concentrates it. The characteristics of the photopolymer material which are used to advantage here are the ability to record at low spatial frequency, high efficiency, self developing nature and ability to record slated gratings [24-25]. This paper studies the recording characteristics of the photopolymer at low spatial frequency and presents a design which will be used to produce the collector device holographically.

Holographic recording in this photopolymer involves creation of a grating in the volume of a layer of polymer by interfering beams of light to produce the required refractive index distribution. The slant angle can be very high, and fringes can even be produced parallel to the plane of the medium (reflection holograms) if required. Higher orders of diffraction are suppressed by the sinusoidal nature of the interference pattern that creates the grating, which is another advantage over binary and multilevel surface gratings. In addition, the refractive index variation is produced in the volume of the material not the surface, so devices can be protected with a plastic cover without affecting performance and/or laminated over one another in stacks. In previous work these photopolymers have been used for the recording of both on and off-axis focusing HOEs, with diffraction efficiencies of $75 \%$ reported for $10 \mathrm{~cm}$ focal length off axis lenses of 100 microns thickness [26].

As mentioned above, multiplexing is also possible in these materials. In previous work 35 gratings with very low diffraction efficiency were multiplexed successfully in an acrylamide based photopolymer [27] for data storage applications. More recently, sets of three and five higher diffraction efficiency gratings and focusing elements have been multiplexed into a single layer of photopolymer [28], at spatial frequencies 450-1700 1/mm and thickness 100-120 microns. This demonstrated the ability to multiplex gratings but the angular range of each individual grating was small.

The aim of the current work is to multiplex/combine a small number of high diffraction efficiency gratings with larger angular working range so that efficient redirection of the incoming light is achieved for incident angles spanning $45^{\circ}$ with a target concentration factor of 20 .

This paper focuses on the first step which is increasing the working range without reducing efficiency for individual gratings made in photopolymer and presents a design for combining the elements into a solar collector. It begins by identifying the optimum spatial frequencies for lower angular selectivity by using the well known Kogelnick theory and then optimizes the grating recording process experimentally for these grating elements at a range of layer thicknesses.

A preliminary design for a device for solar collection is also shown, based on stacked low spatial frequency gratings, Further steps will involve fabrication and testing of the combined device, optimization of the efficiency as well as reducing the number of stacked elements by multiplexing.

\section{THEORY}

The purpose of the diffractive solar collector is to gather sunlight form a large area and direct it onto a smaller area, where it can be converted, for example, using PV cells or thermal conversion. The advantage of using a collector is that the light can be harvested cheaply from a larger area and the energy per unit area on the converter can be increased. As mentioned above, diffraction gratings can be used to change the direction of a light beam very efficiently but they are only efficient over a small range of angles close to the Bragg angle, so they need to be used in combination if they are to be useful in collecting sunlight over most of the day.

The design concept for this diffractive solar collector is a combination of several low spatial frequency photopolymer gratings laminated or stacked together so that light is collected from a broad range of angles. The gratings will form a flat array that will not need to move in order to track the sun's motion across the sky. The low spatial frequency ensures that the angular selectivity of each individual grating is low so that the range of 
angles accepted by each individual grating is maximized and the number of gratings needed in the combined device in minimized.

Figure 1 shows the basic concept of the device. Here, for simplicity, the concept is illustrated for three gratings each having a working range of $6^{\circ}$ and a lens/focusing element also having an working range of $6^{\circ}$ degrees. In practice, a larger number of gratings will be needed if the working range for individual gratings is smaller and/or the desired working range is larger. In the schematic in figure 1 each element is shown separately, but in the practice the gratings will be laminated together without any air gap in order to reduce reflections.

In this example the three gratings are combined to make a device that has a working range of 24 degrees $\left(-3^{\circ}\right.$ to $+21^{\circ}$ ).

If light is incident along or near the normal ( $0^{\circ}$ angular deviation - shown in figure 1 (a)) it will pass through the three gratings A,B,C and be focused by the focusing element D. This element will efficiently focus light over a $6^{\circ}$ range, or $3^{\circ}$ either side of normal incidence. For light that is incident at angles between $3^{\circ}$ and $9^{\circ}$ deviation from normal, the light will be transmitted by A and B but will be on-Bragg for grating C, which will then bend the light though $6^{\circ}$ ensuring its correct alignment for focusing by D (as shown in Figure 1 (b). For light incident with a deviation of $9-15^{\circ}$ from normal, the direction is corrected by $\mathrm{B}$ and then by $\mathrm{C}$ before being focused by $\mathrm{D}$. Similarly for light incident at $15-21^{\circ}$ all three gratings will correct the path of the incident light in sequence before it is focused by D. Gratings A,B and C are all identical in terms of grating spatial frequency and efficiency but the grating slant angle increases moving towards the top of the stack.

The photopolymer materials used in this paper can be laminated to other transparent plastics and/or multiple photopolymer layers, so implementation of this design is more straightforward than in other materials. The high diffraction efficiency is also important in order to minimize losses at multiple gratings. Light incident from higher angles will accumulate more losses because it is diffracted by more of the grating elements. In this paper we focus on optimizing the diffraction efficiency and the angular selectivity using both theory and experiment, so that the individual gratings and /or focusing elements can reach the required parameters.
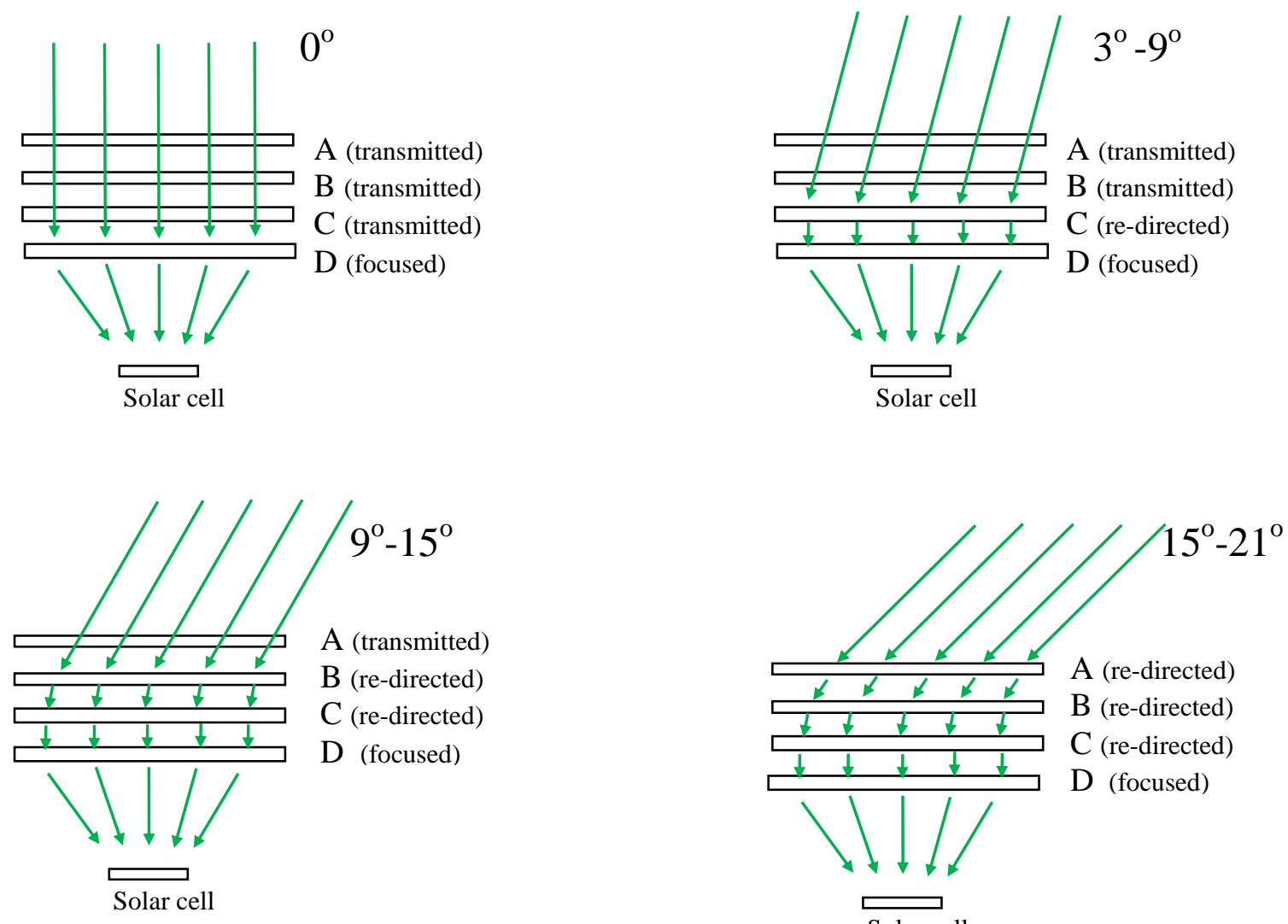

Figure 1 Schematic of the combined diffractive device showing the path of light incident from from a range of angles. 
2.1 Relationship between spatial frequency, hologram thicknesses and angular selectivity

The most important characteristics of a HOE to be used in the above and similar designs for solar concentrators are the angular and wavelength selectivity and the diffraction efficiency. For a given material, efficiency depends on the thickness of the holograms, but increasing the thickness also increases the selectivity, which restricts the range of angles of incidence the device will diffract and also the wavelength range of operation. However, selectivity also depends on the spatial frequency of the grating. At a given thickness, a grating with a lower spatial frequency will have a much greater angular working range. This relationship can be explored using Kogelnik theory [29] which is a widely accepted model used to relate diffraction efficiency and angular selectivity of gratings to the gratings' physical characteristics (thickness, spatial frequency and refractive index modulation).

According to Kogelnik's theory the diffraction efficiency ( $\eta$ ) can be calculated using equation (1), allowing us to model how the diffraction efficiency varies with angle of incidence, near the Bragg angle. This allows us to observe how grating thickness and spatial frequency affect the angular selectivity of an individual grating:

$$
\eta=\frac{\sin ^{2} \sqrt{\left(\xi^{2}+v^{2}\right)}}{\left(1+\frac{\xi^{2}}{v^{2}}\right)}
$$

The parameters $\xi$ and $\mathrm{u}$ are defined as:

$$
\begin{gathered}
\xi=\Delta \theta \frac{k d}{2} \\
v=\frac{\pi n_{1} d}{\lambda \cos \theta}
\end{gathered}
$$

Where $\mathrm{d}$ is the thickness of the grating, $\mathrm{n}_{1}$ is the refractive index modulation; $\lambda$ is the wavelength of the reconstructed beam; $\Delta \theta$ is the deviation from the Bragg angle and $k$ is interference fringe vector.

Figure 2 shows an example of the variation of the diffraction efficiency with the incidence angle for gratings of various thicknesses at a fixed spatial frequency of $250 \mathrm{l} / \mathrm{mm}$. It can be seen in figure 2 that the lowest thickness of $25 \mu \mathrm{m}$ has a wider Full Width Half Maximum (FWHM) of $7.5^{\circ}$ and the highest thickness of $150 \mu \mathrm{m}$ has a narrower FWHM of $1.2^{\circ}$. In use as a solar collector, this will mean that the thinner grating will accept light from a larger range of angles than the thicker one, reducing the number of gratings needed to cover the full angular range of the moving sun.

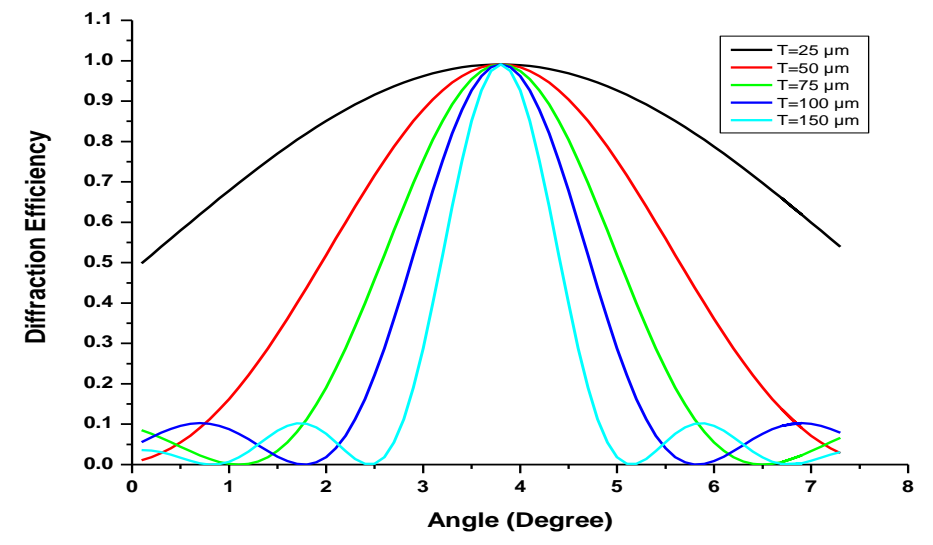

Figure 2 Numerical simulation of the variation of the diffraction efficiency with the incidence angle of gratings of various thicknesses, calculated at a spatial frequency of $250 \mathrm{l} / \mathrm{mm}$. 
The Kogelnik model was then used to determine the angular FWHM of gratings at a range of spatial frequencies and thicknesses typically required to produce efficient gratings in the acrylamide based photopolymer layers. The number of gratings needed in order to collect light from an angle of $45^{\circ}$ was then calculated (based on the FWHM values) and is shown in figure 3.

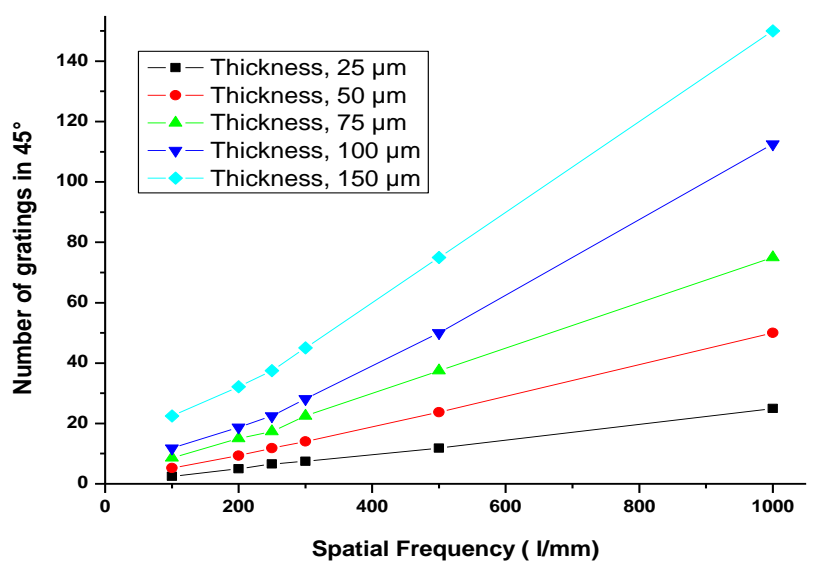

Figure 3 Calculation of the number of multiplexed gratings required to cover a working range of 45 degrees versus spatial frequency. Markers indicate the position of calculated values.

From figure 3 one can see that collection from angles of $45^{\circ}$ is possible with a reasonably small number of gratings for spatial frequencies of 100 to $300 \mathrm{l} / \mathrm{mm}$. Since the lower spatial frequencies provide the most achievable numbers, holographic recording at 100, 200 and 300 l/mm was investigated experimentally.

The materials used here perform well at low spatial frequency but need a minimum of 25-50 microns grating thickness in order to produce good diffraction efficiency gratings, for this reason reducing spatial frequency rather than thickness gives better experimental results.

\subsection{Photopolymerisation}

The basic formulation of a dry photopolymer system contains photoinitiator and monomer/monomers that are hosted by a binder matrix. In the acrylamide-based photopolymer system, photoinitiation is a two-step process. Upon illumination of the photopolymer with light of appropriate wavelength, the sensitizing dye absorbs a photon and reacts with an electron donor to produce free radicals (initiation step). These radicals initiate polymerization in the areas where the light was absorbed. Chain propagation or termination then occurs. During the propagation reaction free radicals interact with monomer molecules or growing polymer chains and thus polymer chains grow. At the termination step two free radicals combine and the polymer chain stops growing. Currently accepted models attribute the recorded modulation of refractive index to the local changes in density and molecular polarizability that accompany polymerization. The magnitude of the refractive index modulation is also dependent on the chain length of the polymer formed, which depends on the rate at which the photons are delivered. Higher recording intensity leads to faster polymerisation rate and the formation of shorter polymer chains, while lower recording intensities result in longer chains [30].

The diffusion models [31-37] predict that the dynamics and properties of the recorded holographic grating (refractive index spatial profile and modulation) are determined by the balance between the polymerization rate and monomer diffusion rate. Both parameters are strongly dependent on the chemical composition of the photopolymer system. Information about their ratio is necessary for the determination of the optimal conditions for holographic recording. At a given spatial frequency, two different regimes of holographic recording can be distinguished with respect to the ratio of the diffusion and polymerization rates. When the polymerization rate is slower than the diffusion rate, the grating profile closely resembles the sinusoidal recording interference pattern and a high saturation value of the refractive index modulation can be achieved. When the monomer diffusion rate is slower than the polymerization rate deviation from the sinusoidal profile of the grating is observed and the diffraction efficiency at saturation is lower. The monomer diffusion rate is characteristic for a given photopolymer system. In a simplified picture, in which the diffusion dependence on the degree of polymerization is not considered, the diffusion time is constant at given spatial frequency. The polymerization rate, on the other hand, depends on the recording intensity. By changing the recording intensity one can control the polymerization rate and in such a way to switch between the two regimes - relatively slow diffusion compared to the polymerization rate at high intensity and relatively fast diffusion when the intensity and the 
polymerization rate are low. Thus in order to achieve high diffraction efficiency at low spatial frequency of recording one expects that relatively low recording intensity will be required.

In materials in which the diffusion of monomers is very slow, the response at low spatial frequency will be very limited even with low intensity of recording. The acrylamide based photopolymer presented in this study is characterised by fast diffusion rate [38] and this allows for the recording of high diffraction efficiency gratings even in the low spatial frequency range of $100-300 \mathrm{l} / \mathrm{mm}$. As it has been already established, such low spatial frequencies are preferable when devices with a larger working range (broad angular and wavelength selectivity curves) are sought.

\section{EXPERIMENT}

\subsection{Materials}

The photopolymer used for the preparation of samples is water-soluble and consists of acrylamide (monomer), N, N' - methylenebisacrylamide (cross linking monomer), triethanolamine (electron donor), polyvinylalcohol (binder) and Erythrosine B (photosensitizer). The photosensitive layer was prepared with composition which has been reported elsewhere [39]. Briefly, the monomers, $0.6 \mathrm{~g}$ acrylamide and $0.2 \mathrm{~g}$ of $\mathrm{N}^{\mathrm{N}} \mathrm{N}^{\prime} \mathrm{Methylene}-$ bisacrylamide and $2 \mathrm{ml}$ of triethanolamine was added to $17.5 \mathrm{ml}$ stock solution of polyvinyl alcohol (PVA) (10\% w/w), the components were mixed well by using a magnetic stirrer and $4 \mathrm{ml}$ of Erythrosin B dye was added finally to sensitise at $532 \mathrm{~nm}$.

Specific volumes of photopolymer solution were spread evenly on a $25 \mathrm{x} 75 \mathrm{~mm}^{2}$ glass plate placed on a levelled surface and dried for 18-24 hours at temperature ranging between 20 -23 degrees and relative humidity ranging $30-40 \%$.

\subsection{Experimental set up}

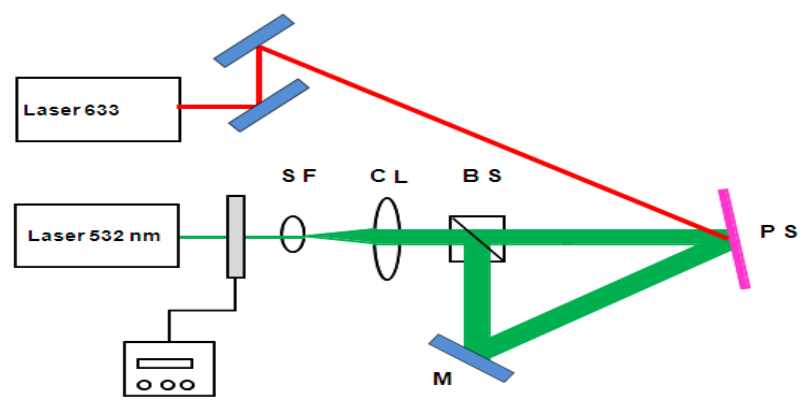

S

Figure 4 Experimental set up: S: shutter, CL: collimating lens, BS: beam splitter, SF: spatial filter, M: mirror, PS: photopolymer sample.

The gratings were recorded in a two-beam holographic optical set up (Fig. 4) using Nd:YVO4 laser (532nm). The spatial frequency was altered by changing the angle between the beams used to record the gratings. The Bragg equation (4) was used to calculate the angle between the two interfering beams:

$$
\lambda=2 \Lambda \sin \theta
$$

Where $\lambda$ is recording wavelength, $\Lambda$ is fringe spacing; $\Theta$ is half of the angle between the two interfering beams.

In this study gratings were recorded in various layers thicknesses with spatial frequency of $100 \mathrm{l} / \mathrm{mm}, 200 \mathrm{l} / \mathrm{mm}$ and $300 \mathrm{l} / \mathrm{mm}$ and the intensity of recording beam was varied between $1-3 \mathrm{~mW} / \mathrm{cm}^{2}$. A vertically polarized 633 $\mathrm{nm}$ laser beam (He- $\mathrm{Ne}$ ) was used as a probe beam. During recording this was incident at the Bragg angle for the particular spatial frequency in order to monitor the diffraction efficiency of the grating in real time. In order to characterize the diffracted intensity dependence on the incident angle of the probe beam, the grating was placed on a rotational stage (Newport, ESP 300) and an optical power meter (Newport 1830-C) was used to record the intensity of the diffracted beam. The data was recorded by a data acquisition card and LabVIEW program. In order to determine the diffraction efficiency of the recorded gratings/lenses, equation (5) was used:

$$
\eta=\frac{I_{d}}{I_{\text {in }}} \times 100
$$

$6 \mid \mathrm{P}$ a g e 
Where $\mathrm{I}_{\mathrm{d}}$ is diffracted beam intensity, $\mathrm{I}_{\text {in }}$ is incident (probe beam) intensity and $\eta$ is diffraction efficiency.

\section{RESULTS AND DISCUSSION}

In this paper the experimental work largely focuses on investigating the best way to record high diffraction efficiency gratings at these low spatial frequencies. In order to study this, transmission gratings were recorded at each of three spatial frequencies for a number of sample thicknesses and at various recording intensities. The diffraction efficiency of each recorded grating was measured in real time. A summary of the results is presented below.

\subsection{Comparison of the diffraction efficiency of transmission gratings recorded at different spatial frequencies}

The diffraction efficiency of gratings were measured during holographic recording and compared for polymer layers of thicknesses ranging from $50 \mu \mathrm{m}$ to $150 \mu \mathrm{m}$ at spatial frequency $300 \mathrm{l} / \mathrm{mm}, 200 \mathrm{l} / \mathrm{mm}$ and $100 \mathrm{l} / \mathrm{mm}$. Exposure intensity was $1 \mathrm{~mW} / \mathrm{cm}^{2}$. The results for all three spatial frequencies are shown in Figure 5. High efficiency achieved at low thickness and low spatial frequency is of most interest as these are the gratings that will have the maximum working range. The maximum diffraction efficiency observed here was $80 \%$ and this was achieved at spatial frequencies of 200 and $300 \mathrm{l} / \mathrm{mm}$ for layer thicknesses $75 \mu \mathrm{m}$ and above. $50 \mu \mathrm{m}$ layers achieved $75 \%$ efficiency at a spatial frequency of $300 \mathrm{l} / \mathrm{mm}$. For layers of thickness of $150,100 \mu \mathrm{m}$ and even 75 $\mu \mathrm{m}$ over-modulation was observed at longer exposures indicating that the refractive index of the grating has grown beyond the value that corresponds to the first maximum in diffraction efficiency at the particular thickness used.

From these results we observe that the very low spatial frequency $100 \mathrm{l} / \mathrm{mm}$ does not achieve high diffraction efficiency, but 200 and $300 \mathrm{l} / \mathrm{mm}$ perform well. We also observe that thickness as low as $50 \mu \mathrm{m}$ can achieve high efficiency at spatial frequency of $300 \mathrm{l} / \mathrm{mm}$. The low diffraction efficiencies observed at $100 \mathrm{l} / \mathrm{mm}$ are due to a combination of reduced diffusion and loss of light into higher orders because the grating is now behaving less like a thick grating. For ease of comparison the y-axes on each of the three graphs in figure 5 are given the same scale and range.

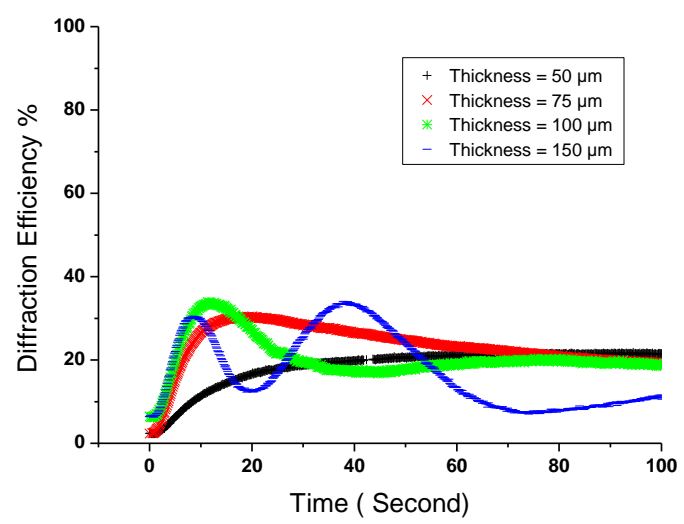

(a)

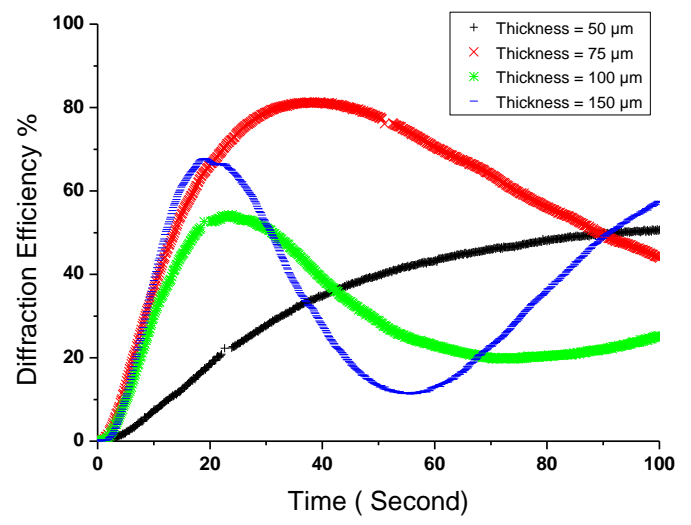


(b)

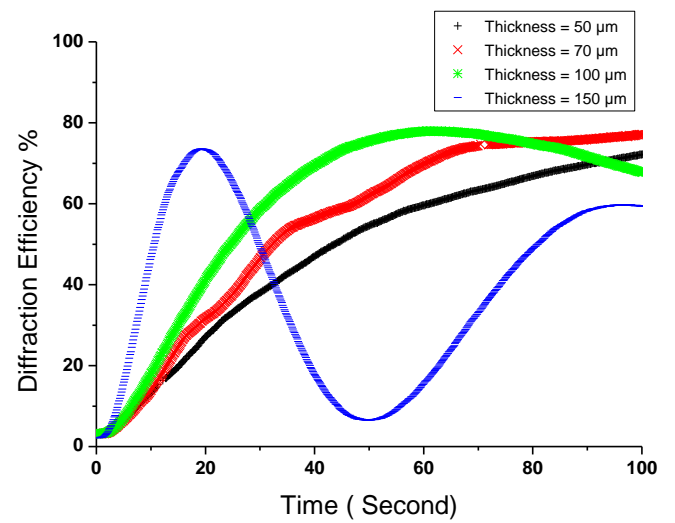

(c)

Figure 5 Real time measurements of the diffraction efficiency of gratings recorded in polymer layers of various thickness;

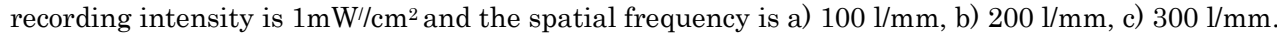

The next study examined how the diffraction efficiency evolution during recording depends on the recording intensity. The purpose of this study was to identify the maximum diffraction efficiency achievable at each spatial frequency by optimizing the recording conditions.

\subsection{Dependence of diffraction efficiency on recording intensity at spatial frequency of $100 \mathrm{l} / \mathrm{mm}$}

In order to determine the optimum recording intensity at each spatial frequency, experiments were carried out at different intensities. Transmission gratings were recorded in the photopolymer layers using a recording intensity which was varied from 1-3 $\mathrm{mW} / \mathrm{cm}^{2}$.

The results presented in figure 6 show the relationship between first order diffraction efficiency of the transmission gratings and recording intensity, for a spatial frequency of $100 \mathrm{l} / \mathrm{mm}$, in layers of thicknesses of $50 \mu \mathrm{m}$ and $75 \mu \mathrm{m}$. From the graphs it can be clearly seen that the diffraction efficiency achieved at each exposure is not highly dependent on recording intensity at this particular spatial frequency. This observation could be explained by the large fringe spacing, $10 \mu \mathrm{m}$. Both $1 \mathrm{~mW} / \mathrm{cm}^{2}$ and $2 \mathrm{~mW} / \mathrm{cm}^{2}$ lead to similar values of the maximum achievable diffraction efficiency. Recording with $3 \mathrm{~mW} / \mathrm{cm}^{2}$ intensity leads to a slight decrease in the diffraction efficiency. The maximum diffraction efficiency achieved at $100 \mathrm{l} / \mathrm{mm}$ was about $35 \%$.

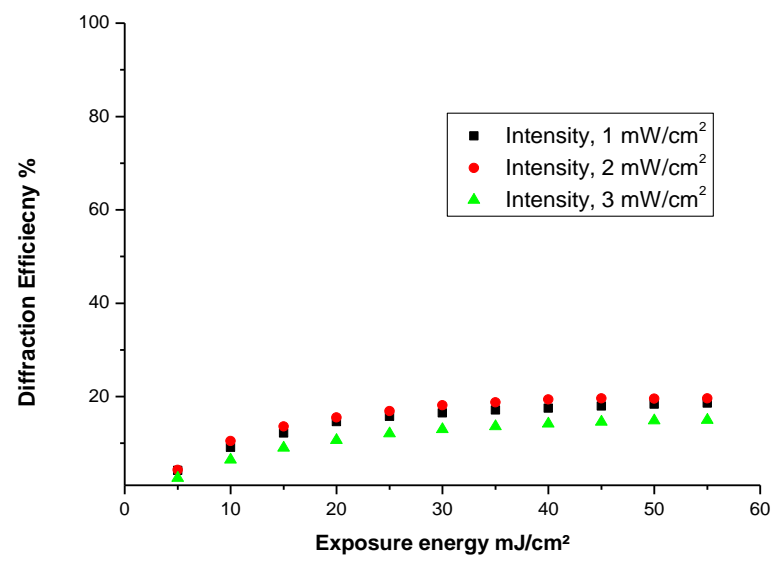

(a)

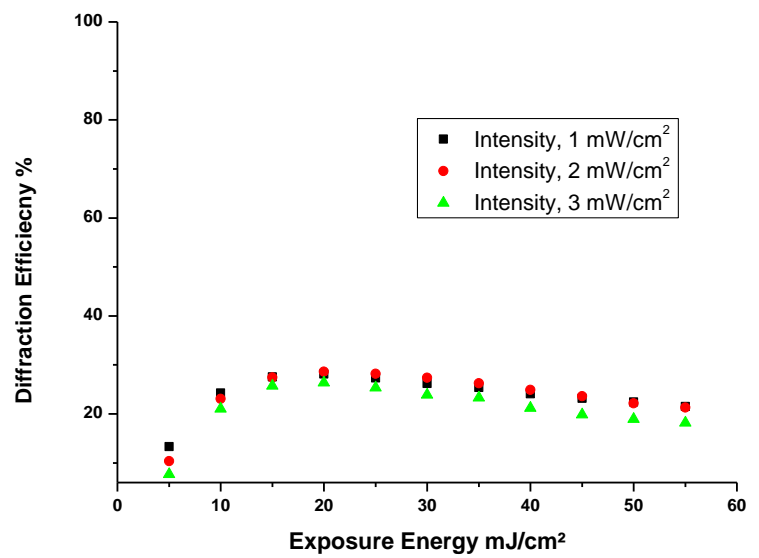

(b)

Figure 6 Measured diffraction efficiency vs. Exposure energy for transmission gratings with intensity from 1-3 $\mathrm{mW} / \mathrm{cm}^{2}$ at spatial frequency of $100 \mathrm{l} / \mathrm{mm}$ investigated for sample thicknesses of a) $50 \mu \mathrm{m}$, b) $75 \mu \mathrm{m}$. 
4.3 Dependence of diffraction efficiency on recording intensity at spatial frequency of $200 \mathrm{l} / \mathrm{mm}$

The experiment was repeated for $200 \mathrm{l} / \mathrm{mm}$. At this spatial frequency, the maximum diffraction efficiency obtained for this particular sample thickness was about $80 \%$. This was obtained at a recording intensity of $1 \mathrm{~mW} / \mathrm{cm}^{2}$. The results reveal that there is intensity dependence at this spatial frequency. As has been observed in previous work, delivery of the same energy over a longer time period allows longer polymer chains to be formed and increases the diffraction efficiency achieved. This is because diffusion rate is high relative to the rate of polymerization. This is easier to observe as the spatial frequency increases because the smaller fringe spacing allows easy diffusion of monomer into the bright fringe regions from the dark fringe regions.

Figure 7 presents the diffraction efficiency dependence on intensity at spatial frequency of $200 \mathrm{l} / \mathrm{mm}$ for sample thicknesses of $50 \mu \mathrm{m}$ and $75 \mu \mathrm{m}$.

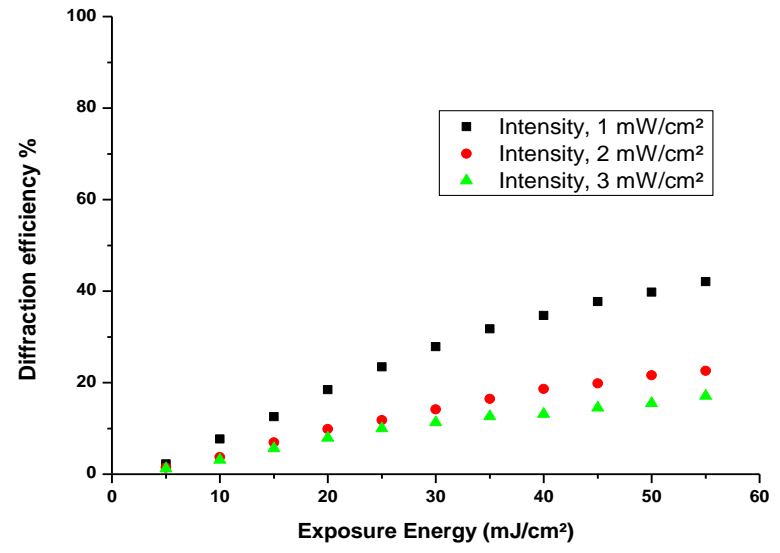

(a)

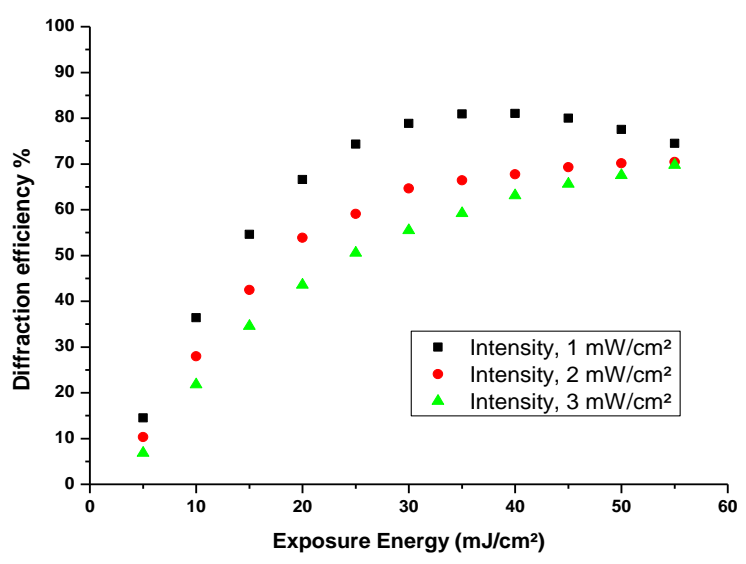

(b)

Figure 7 Measured diffraction efficiency vs. Exposure energy for transmission gratings recorded with intensity between 1-3 $\mathrm{mW} / \mathrm{cm}^{2}$ at spatial frequency of $200 \mathrm{l} / \mathrm{mm}$ was investigated for sample thicknesses of a) $\left.50 \mu \mathrm{m}, \mathrm{b}\right) 75 \mu \mathrm{m}$.

4.4 Dependence of diffraction efficiency on recording intensity at spatial frequency of $300 \mathrm{l} / \mathrm{mm}$

The experiment was repeated for $300 \mathrm{l} / \mathrm{mm}$. The results are shown in Figure 8. Again, the maximum diffraction efficiency is achieved at lower recording intensities. As in the case of recording at $200 \mathrm{l} / \mathrm{mm}$, a clear dependence of diffraction efficiency on recording intensity was observed in both layers of 50 and $75 \mu \mathrm{m}$ thickness. Recording at lower intensity lead to higher diffraction efficiency. At lower intensity the polymerisation process is slower and thus the monomer molecules have enough time to diffuse from dark to bright regions and contribute to the final refractive index modulation.

The spatial frequency of $300 \mathrm{l} / \mathrm{mm}$ and $200 \mathrm{l} / \mathrm{mm}$ will be used for further study in this application where the HOE lenses with relatively high diffraction efficiency can be investigated. 


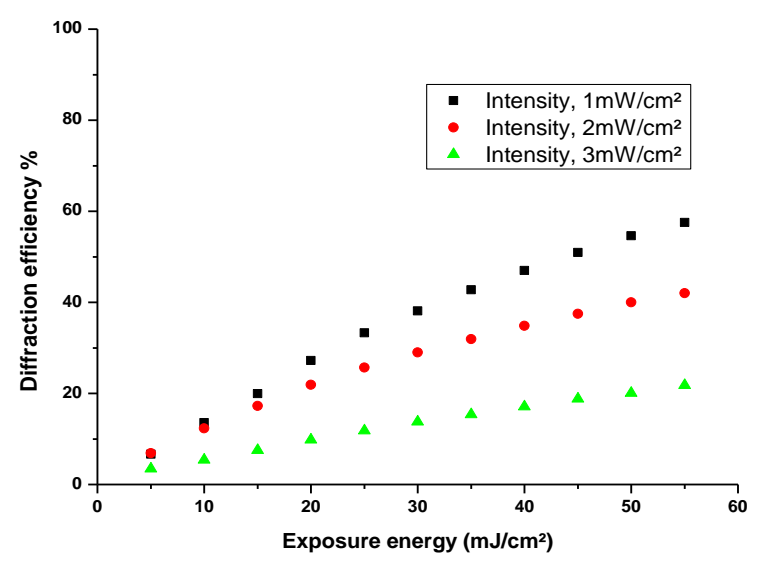

(a)

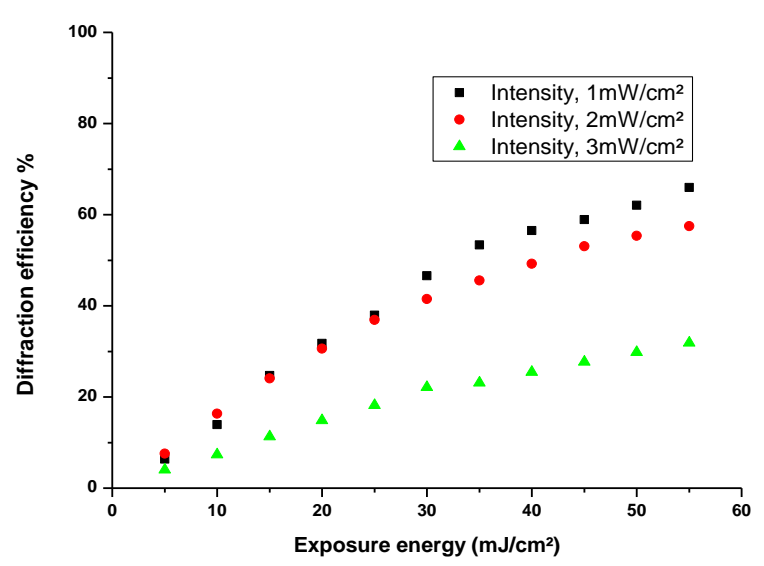

(b)

Figure 8 Measured diffraction efficiency vs. Exposure energy for the transmission gratings with various intensities between 1$3 \mathrm{~mW} / \mathrm{cm}^{2}$ at spatial frequency of $300 \mathrm{l} / \mathrm{mm}$ was investigated for sample thicknesses of a) $\left.50 \mu \mathrm{m}, \mathrm{b}\right) 75 \mu \mathrm{m}$.

4.5 Angular and wavelength dependence of diffraction efficiency in recorded HOES

The variation of diffraction efficiency with angle of incidence for a grating with spatial frequency of 300 lines/mm and thickness 50 microns is shown in Figure 9.

The solid line shows the theoretical relationship predicted using the equations in section 1 . The experimental data matches closely with this. From the graph we can see that the FWHM is approximately 3 degrees.

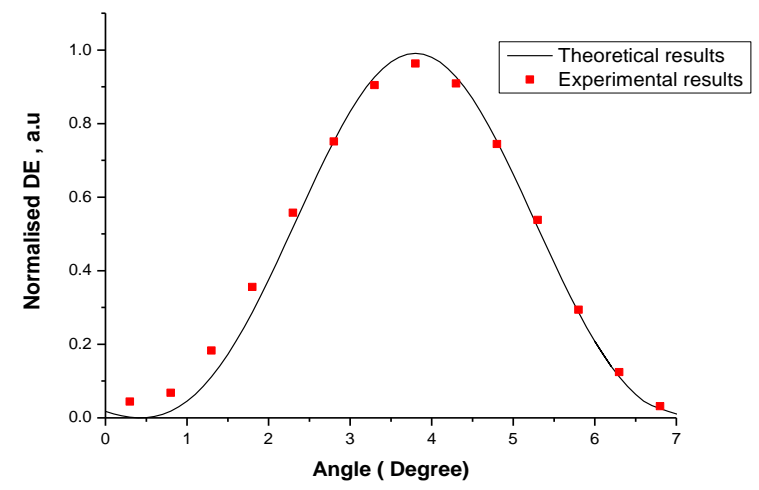

Fig. 9 Experimental and theoretical angular selectivity curves for a grating with spatial frequency of $300 \mathrm{l} / \mathrm{mm}$ recorded in layers with thickness of $50 \mu \mathrm{m}$.

A range of HOE's with an off-axis focusing effect were also recorded in order to demonstrate focusing elements with low spatial frequency and broad working range. The example shown has a central spatial frequency of 300 $\mathrm{l} / \mathrm{mm}$. The experimental setup was the same as that described above for recording gratings except that a lens was placed in one of the recording beams. The focal length of the recorded lens element was $5 \mathrm{~cm}$ and the element diameter was approximately $0.9 \mathrm{~cm}$. The diffraction efficiency of each $\mathrm{HOE}$ was determined using equation (5), by measuring the diffracted beam's intensity close to the focal point.

As real-time monitoring of the diffraction efficiency was not possible in this instance, the diffraction efficiency was determined for a series of HOE's made using different exposure times at a recording intensity of $1 \mathrm{~mW} / \mathrm{cm}^{2}$. $50 \mu \mathrm{m}$ layers were used in this experiment. Figure 10 (a) shows the diffraction efficiency versus the exposure time; it is observed that the diffraction efficiency reaches a maximum of about $75 \%$ after about 100 seconds. Comparison with the real-time measurements for gratings of similar thickness and spatial frequency shows that recording is very similar for gratings and focusing elements. The variation of diffraction efficiency with angle of 
incidence is also shown in Figure 10 (b). The FWHM angular selectivity of the lenses is shown to be approximately 3 degrees. For comparison, the simulated Bragg curve for a 300 line/mm grating of 50 microns thickness is also shown on the graph. The fit is not exact because a focusing lens has a range of spatial frequencies around a central frequency, rather than a single spatial frequency.

Figure 11 is a photograph of one of the photopolymer focusing elements recorded as described above. The photopolymer is sandwiched between two protective plastic layers and a single off-axis focusing element is visible towards the centre of the layer. The proposed device will contain arrays of such elements and multiple layers.

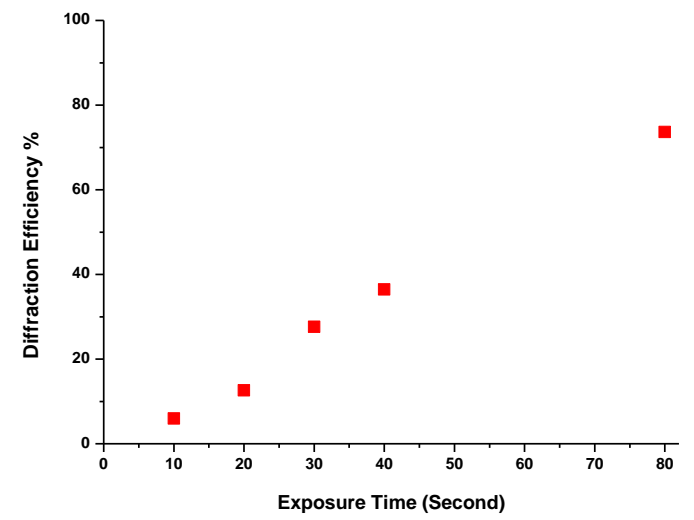

(a)

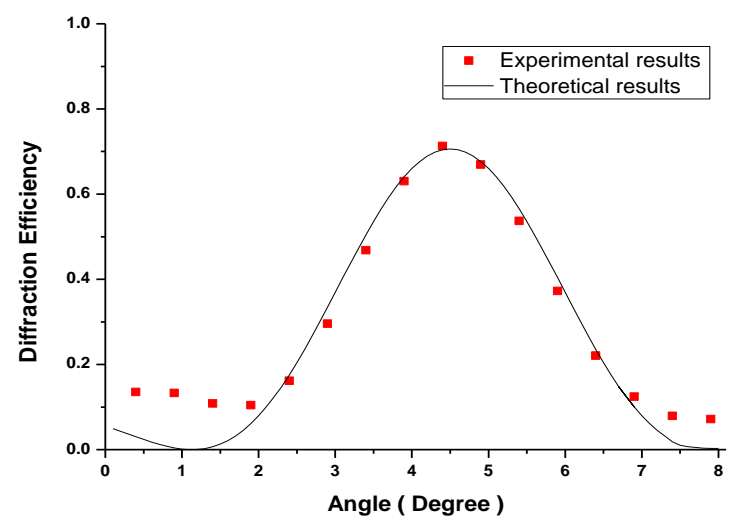

(b)

Figure 10 (a) Diffraction efficiency vs. exposure time for HOE's (b) Diffraction efficiency vs. angle for HOE's at spatial frequency of $300 \mathrm{l} / \mathrm{mm}$ and recording intensity of $1 \mathrm{~mW} / \mathrm{cm}^{2}$ was investigated for sample thickness of $50 \mu \mathrm{m}$.

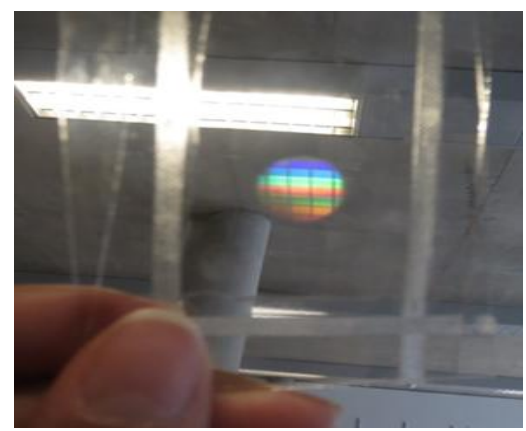

Figure 11 Photo taken through holographic lenses recorded in acrylamide photopolymer

\subsection{Wavelength selectivity of focusing $\mathrm{HOES}$}

Volume gratings are generally much less selective for wavelength than for angle. However, in order to confirm the there is a reasonable working range for these devices the wavelength selectivity of recorded HOE's was determined using an integrating sphere. A white light source (Avantes, AvaLight-Hal-S) was used as a probe beam in these experiments. The diffraction efficiency of focusing HOEs at different wavelengths (460 nm, 532 $\mathrm{nm}$ and $640 \mathrm{~nm}$ ) was collected by using the integrating sphere (Avantes, AvaSphere-50-REFL) coupled to optical fibre connected to a spectrometer (Avantes, AVASPEC 2048-USB2) which was used to monitor the photon count rate of the integrating sphere. 
In this case the HOE lenses had been recorded with intensity of $2.5 \mathrm{~mW} / \mathrm{cm}^{2}$ in $30 \mu \mathrm{m}$ layers and had a central spatial frequency of $300 \mathrm{l} / \mathrm{mm}$. The diffraction efficiency $(\eta)$ of recorded lenses at ach different wavelength can be estimated by measuring the intensity of zero order without $\mathrm{HOE}$ lens $\left(\mathrm{I}_{0}\right)$ and with $\mathrm{HOE}$ lens ( $\left.\mathrm{I}_{0}{ }^{\prime}\right)$, at the optimum Bragg position by using the equation (6):

$$
\eta=\frac{I_{0}-\left(I_{0}{ }^{\prime}\right)}{I_{0}} \times 100
$$

The results in figure 12 shown that the HOE lenses achieved a maximum diffraction efficiency of about $54 \%$ at wavelength of $460 \mathrm{~nm}, 60 \%$ at wavelength of $540 \mathrm{~nm}$ and $41 \%$ at wavelength of $633 \mathrm{~nm}$. . The sample tested was $30 \mu \mathrm{m}$ thick, so the peak diffraction efficiencies are lower than in the examples above, however, it is clear that the working range covers a significant portion of the visible spectrum for these gratings.

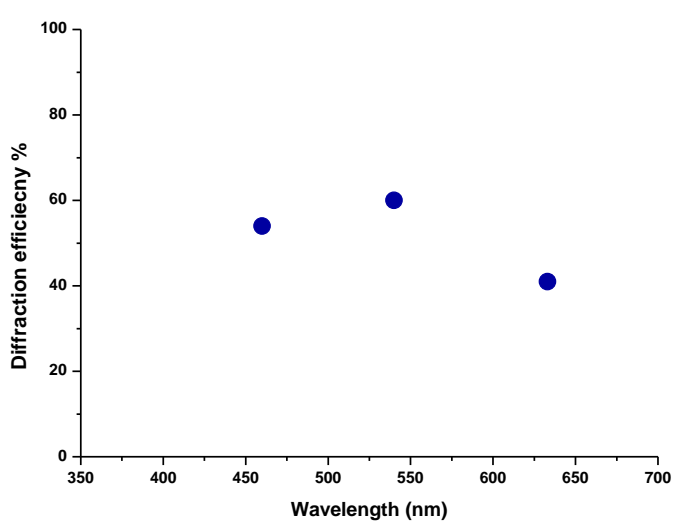

Figure 12 Experimental data of the Diffraction efficiency vs. Wavelength for the HOE lenses recorded at intensity of $2.5 \mathrm{~mW} / \mathrm{cm} 2$ at various wavelengths of $460 \mathrm{~nm}, 540 \mathrm{~nm}, 633 \mathrm{~nm}$ respectively was investigated for sample of $30 \mu \mathrm{m}$ thickness.

\subsection{Concentration factor of HOEs devices}

The target diffraction efficiency for this application will be $90 \%$ for each grating element (after reflection losses) and $80 \%$ for the lens element. The $80 \%$ diffraction efficiency reported above is not corrected for reflection or absorption losses, but was measured by monitoring the diffracted beam during recording and calculating the intensity ratio to the incident beam; $80 \%$ of the incident intensity is in the diffracted /focused beam. Recent results have confirmed that $90 \%$ efficiency can be achieved with careful optimization of recording and on line monitoring of the diffraction efficiency growth.

In estimating the overall efficiency of the device we can assume $4 \%$ reflection losses at the top and bottom surfaces only, because the layers will be laminated together without air gaps.

For a 4-element device of the type proposed in the introduction we therefore expect a $10 \%$ reduction at each individual element. This will mean that at worst roughly half the incident intensity will be in the focused beam for this combined element. This estimate is for beams diffracted at all three gratings as well as the focusing element. Therefore in order to reach a target concentration factor of 20 for the combined device we aim for a concentration factor of 40 for the focusing element. However, losses are lower for the lower angles of incidence because the beams are not diffracted at the upper layers.

Taking the example of a diffractive lens element with approximately $1 \mathrm{~cm}$ radius and focal length of $5 \mathrm{~cm}$, readily achievable in the lab, we can estimate typical concentration factors. For example, if a PV cell is located $0.75 \mathrm{~cm}-1.0 \mathrm{~cm}$ from the focal point $(4.0 \mathrm{~cm}-4.25 \mathrm{~cm}$ below the lens element), a concentration factor of between 25 and 44 would be achieved by the lens . 


\section{CONCLUSIONS}

Modeling confirmed that lower spatial frequencies are more suitable for capturing light over a wide range of angles. At the thicknesses most commonly used in acrylamide based photopolymers, spatial frequencies as low as a few hundred lines per millimeter are necessary in order to keep the number of multiplexed or stacked gratings used in a solar collector low. Experimental work to optimize the holographic recording characteristics of the photopolymer material at these low spatial frequencies was presented. It showed that low intensity of recording produces higher efficiency gratings and focusing elements. Maximum diffraction efficiencies of $75 \%$ and $80 \%$ were observed in photopolymer layers of $50 \mu \mathrm{m}$ and $75 \mu \mathrm{m}$ thickness at these spatial frequencies. This means that $80 \%$ of the incident light was measured in the diffracted beam with no correction for reflection, absorption or other losses. Focusing elements were observed to have very similar performance to gratings. Typical angular range (FWHM) were $3^{\circ}$.

The advantage of using thinner layers and lower spatial frequency of recording in this application is the larger angular and wavelength range of the optical component. Future work will focus on improvement of diffraction efficiency, fabrication of multiple-element devices, and characterization of their performance.

\section{ACKNOWLEDGEMENTS}

The authors would like to acknowledge the School of Physics and the FOCAS Institute for use of equipment and facilities at the Dublin Institute of Technology.

\section{REFERENCES}

[1] Marshall AJ, Young DS, Blyth J, Kabilan S and Lowe CR, 2004 Metabolite-sensitive holographic biosensors Anal. Chem 76(5) 1518-1523.

[2] Bhatta D, Christie G., Madrigal-González B, Blyth J and Lowe CR 2007 Holographic sensors for the detection of bacterial spores Biosensing and. Bioelectronics 23(4) 520-527.

[3] Naydenova I, Jallapuram R, Toal V, Martin S, 2009 A visual indication of environmental humidity using a colour changing hologram recorded in a self-developing photopolymer Appl. Phys. Lett 92(3) 031109-031109-3.

[4] Naydenova I, Jallapuram R, Toal V, Martin S, 2009 Characterisation of the humidity and temperature responses of a reflection hologram recorded in acrylamide-based photopolymer Sensors Actuat. B-Chem 139(1) 35-38.

[5] Naydenova I, Martin S, Toal V 2009 Photopolymers - beyond the standard approach to photosensitisation. J.Eur. Opt. Soc 409042.

[6] Leite E, Naydenova I, Mintova S, Leclercq L, Toal V 2010 Photopolymerisable Nanocomposites for Holographic Recording and Sensor Application Appl. Opt.49(19) 3652-3660.

[7] Leite E, Babeva T, Ng EP, Toal V, Mintova S, and Naydenova I 2010 Optical Properties of Photopolymer Layers Doped with Aluminophosphate Nanocrystals Journal of Phys. Chem. C, 114(39) 16767-16775.

[8] Hernandez-Montes MdS, Furlong C, Rosowski JJ, Hulli N, Harrington E, Cheng JT, Ravicz ME and Santoyo FM 2009 Optoelectronic holographic otoscope for measurement of nano-displacements in tympanic membranes Journal of Biomedical Optics 14(3) 034023.

[9] Battey D, Slater J and Wludyka R, 1993 Axial transmissive f/1.8 imaging raman spectrograph with volumephase holographic filter and grating Applied Spectroscopy 47(11) 1913-1919.

[10] Sudarshanam V and Pappu S 1992 Holographic Optical Elements based single-mode hybrid fiber optic interferometer for realizing zero-order fringe Fiber and Integrated Optics 10(1) 71-83. 
[11] Trolinger JD, Lal RB and Batra AK 1991 A Holographic instrumentation for monitoring crystal-growth in space Optical Engineering, 30(10): p. 1608-1614.

[12] Gu T, Zhu M, Fang S 2005 Three-dimensional imaging system with holographic scanner and auxiliary reflectors Holography, Diffractive Optics and Applications II Proc SPIE 5636 ed. Yunlong Sheng, Dahsiung Hsu, Chongxiu Yu, Byoungho Lee 387-391.

[13] Cheng C 1998 High-performance horizontal side scanner using holographic technology Input/Output and Imaging Technologies SPIE Proc. 3422 (Taipei, Taiwan June 18 1998) ed. Yusheng Tim Tsai; Teh-Ming Kung; Jan Larsen 257-262.

[14] Herzig HP and Dandliker R 1988 Holographic optical scanning elements with minimum aberrations Appl Opt 27 4739-4746.

[15] Huang G, Pan L, Xu D, Pei J, Jia H 1996 Holographic elements for CD pickup-to-play DVD Proc Optical Recording, Storage, and Retrieval Systems SPIE Proc 2890 38-42.

[16] Lee WH 1989 Holographic optical head for compact disk applications Opt Eng 28 650-653.

[17] Bloss WH, Griesinger M and Reinhardt ER 1982 Dispersive concentrating systems based on transmission phase holograms for solar applications Appl. Opt 21 3739-3742.

[18] Zhang D, Castro JM and Kostuk RK 2011 One-axis tracking holographic planar concentrator system Journal of Photonics for Energy 19477988 (doi:10.1117/1.3590943).

[19] Ranjan R, Ghosh A, Nirala A.K, Yadav H.L 2013 Optimization of processing parameters of holographic concentrator for maximum efficiency operation in PV system Publisher Wseas LLC, Volume 8 of Energy, environmental and structural engineering series, ISSN 2227-4359 (ISBN 1618041754, 9781618041753).

[20] Ludman J 1982 Holographic solar concentrator Applied Optics 21(17) 3057-3058.

[21] Zhang YW, Ih CSI, Yan HF, Chang MJ 1988 Photovoltaic concentrator using a holographic optical element Applied. Optics 27 3556-3560.

[22] Ren X, Liu S, Zhang X, Chen X 2007 Fabrication of holographic Fresnel lens used as solar concentrator Holography and Diffractive Optics III SPIE Proc. 6832 (Beijing, China, 12-14 November 2007) ed. Yunlong Sheng, Dahsiung Hsu, Chongxiu Yu 1-7.

[23] Shaji Sam T L, Vadivelan V, Ajith Kumar PT 2007 A multi-beam holographic light concentrator for solar applications Holography and Diffractive Optics III SPIE Proc. 6832 , 68321E (Beijing, China, 12-14 November 2007) ed. Yunlong Sheng, Dahsiung Hsu, Chongxiu Yu 114.

[24] Gallego S, Neipp C, Ortuno M, Marquez A, Belendes A and Pascual I 2003 Diffusion-based model to predict the conservation of gratings recorded in poly(vinyl alcohol) acrylamide photopolymer Appl. Opt. 42(29) 58395845 .

[25] Martin S, Leclere P, Renotte Y, Toal V and Lion Y, 1994 Characterisation of an acrylamide-based dry photopolymer holographic recording material Optical Engineering 33(12) 3942-3946.

[26] Martin S, Feely CA, Sheridan JT, Toal V 1998 Applications of a self-developing photopolymer material: holographic interferometry and high-efficiency diffractive optical elements, Holographic Materials IV, SPIE photonics west, San Jose Proc. SPIE 3294 (60-70).

[27] Sherif H, Naydenova I, Martin S, McGinn C and Toal V 2005 "Characterization of an acrylamide-based photopolymer for data storage utilizing holographic angular multiplexing”, J. Opt. A. Pure Appl. Opt. 7(5), 255260.

$14 \mid \mathrm{P}$ a g e 
[28] Naydenova I, Akbari H, Dalton C, Ilyas MYM, Wei CPT, Toal V and Martin S, 2012 Photopolymer Holographic Optical Elements for application in solar energy concentrators Holography-Basic Principles and Contemporary Applications InTech Ed. Mihaylova E p130 (http://dx.doi.org/10.5772/55109).

[29] Toal V 2011 Introduction to Holography (Boca Raton, Florida) CRC Press, Taylor \& Francis Group. p 472.

[30] Tomlinson W and Chandross E 1980 Organic Photochemical Refractive Index Image Recording Systems Adv. Photochem. 12, John Wiley Interscience, Ed. Pitts TN et al. pp. 201-280.

[31] Zhao G and Mourolis P 1994 Diffusion model of hologram formation in dry photopolymer materials. J. Mod. Opt. 41 1929-1939.

[32] Moreau V, Renotte Y and Lion Y 2002 Characterisation of DuPont photopolymer: determination of kinetic parameters in a diffusion model Appl. Opt 41 3427-3435.

[33] Colvin VL, Larson RG, Harris AL and Schilling ML 1997 Quantitative model of volume hologram formation in photopolymers J. Appl. Phys 81 5913-5923.

[34] Kwon JH, Hwang HC and Woo KC 1999 Analysis of temporal behaviour of beams diffracted by volume gratings formed in photopolymers J. Opt. Soc. Am. B 16 1651-1657.

[35] Piazzolla s and Jenkins BK 2000 First harmonic diffusion model for holographic grating formation in photopolymers J. Opt. Soc. Am. B17 1147-1157.

[36] Lawrence JR, O.Neill FT and Sheridan JT 2002 Adjusted intensity nonlocal diffusion model of photopolymer grating formation J. Opt. Soc. Am. B19 621-629.

[37] Babeva T, Naydenova I, Mackey D, Martin S and Toal V 2010 Two-way diffusion model for short-exposure holographic grating formation in acrylamidebased photopolymer JOSA B, 27(2) 197- 203.

[38] Naydenova I, Martin S, Jallapuram R, Howard R and Toal V 2004 Investigations of the diffusion processes in a self-processing acrylamide-based photopolymer system Applied Optics 43 2900-5.

[39] Martin S, Feely CA and Toal V 1997 Holographic recording characteristics of an acrylamide-based photopolymer Applied Optics 36 (23) 5757-5768. 